\title{
Fabric Defect Detection in Textile Images Using Gabor Filter
}

\author{
TanveerSajid ${ }^{1}$, Baqir Ali ${ }^{2}$ \\ ${ }^{1,2}$ (Department of Electronic Engineering, International Islamic University Islamabad, Pakistan)
}

\begin{abstract}
Fabric defect detection is one of the most important and challenging field in textile industry. In recent times 2-D Gabor filter technique can be used for defect detection in images. This paper aims at investigating methods for solving the problem of fabricdefectdetection.The work focuses on two aspects. First detect the defects in images using Gabor filter and Morphology technique. Second calculate the defective areas in images by calculating black and white pixels in the images and at the end compare the effective segmented area of proposed algorithm with morphology technique. Fabric images are used in evaluation and the experimental results obtained further confirm the designed algorithm achieved a high detection success rate.
\end{abstract}

Keywords: Defect detection, segmentation, Gabor filter, morphology, pixels.

\section{Introduction}

In the past two decades during which computer vision based inspection has become one of the most important application areas. Fabric industry is one of the most important field in identifying defects or flaws. But these days lot of work can be done on to remove defects in images of fabric in fabric industry and calculate defective areas in fabric images more precisely.

Defect detection techniques have been classified in four categories: Statistical, Spectral, Model based and filter based. In statistical approaches important techniques like GLCM, Local binary pattern, autocorrelation can be used for defect detection. Features like energy, entropy, contrast and correlation can determine with the help of GLCM. It's based on repetitive occurrence of different grey level configurations in a texture. GLCM method can face number of shortcomings [11]. There is no generally accepted solution for optimizing the displacement vector. Local binary pattern can be used for detection in local image contrast. LBP can chooses the gray level of the centre pixel of the sliding window as a threshold for surrounding neighborhood pixels [12].In spectral approaches techniques like discrete Fourier transform, windowed Fourier transform and wavelet transform can be used for defect detection. Windowed Fourier transform can adopted for identification of defects that occurs locally both in spatial and frequency domain. In model based approach GMRF can use for defect detection. In GMRF image can split into small window in inspection. Then a likely hood estimator test can be used for detecting defect in window occurs or not. In filter based method techniques like spatial and frequency domain can be used. Ring and wedge filters are mostly used as frequency domain filters. In spatial domain mostly used filters like sobel, canny, laplacian, deriche, law filters can be successively used for defect detection.

The Fourier transform has the property to only deals with frequency component in the signal. It is not be able to localize defects in spatial domain. Gabor transform localize the defects both in spatial domain and frequency domain. Gabor filter forms very flexible power spectrum When window function become Gaussian than windowed Fourier transform became well defined Gabor filter bank approach has broadly studied in visual inspection and at the end compare the results of proposed algorithm with morphology technique.

\section{Method}

\section{Gabor filter}

\subsection{Two dimensional Gabor filter banks}

Two dimensional Gabor filter can be written

$g(x, y, \lambda, \theta, \psi, \varphi, \gamma)=\exp \left(-\frac{x^{\prime 2}+\gamma^{2} y^{\prime 2}}{2 \sigma^{2}}\right) \cos \left(2 \pi \frac{x^{\prime}}{\lambda}+\psi\right)$

where

$$
\begin{aligned}
& x^{\prime}=x \cos \theta+y \sin \theta \\
& y^{\prime}=-x \sin \theta+y \cos \theta
\end{aligned}
$$

In this equation (1) $\lambda$ represents the wavelength of the cosinefactor, $\theta$ represents the orientation of the normal to the parallelstripes of a Gabor function in degrees, $\psi$ is the phase offset indegrees, and $\gamma$ is the spatial aspect ratio and specifies the elliptically of the support of the Gabor function, and $\sigma$ is the standard deviation of the Gaussian determines the (linear) sizeof the receptive field [16].The parameter $\lambda$ is the wavelength and $f=1 / \lambda$ is the spatial frequency of the cosine factor. The ratio $\sigma / \lambda$ determines the spatial 
frequency bandwidth of simple. The half -response spatial frequency bandwidth $b$ (in octaves) and the ratio $\sigma / \lambda$ are related in equation (2) as follows:

$$
b=\log _{2} \frac{\frac{\sigma}{\lambda} \pi+\sqrt{\frac{\ln 2}{2}}}{\frac{\sigma}{\lambda} \pi-\sqrt{\frac{\ln 2}{2}}}
$$

In equation (1) $\psi=0^{0}$ and $\psi=90^{\circ}$ returnsthe real part and the imaginary part of Gabor filter respectively.

\section{$1.2 \quad$ Feature extraction}

We use sigmoid function for feature extraction which can be written as given below,

$$
\tanh (\alpha t)=\frac{1-e^{-2 \alpha t}}{1+e^{-2 \alpha t}}
$$

This saturates the output of the filter and than use Gaussian smoothing function for image smoothness [10]. This can be written as ,

Where sigma represents the standard deviation, determines the size and shape of the respective field depictedin equation (4).

\subsection{Clustering}

The final step of the algorithm is clustering the pixels into multiple numbers of clusters represents the texture regions. In thesis work $\mathrm{k}$ means algorithm can be used for segmentation process due to its simplicity [18]. $\mathrm{k}$ means algorithm starts with recognition of the centroids of $\mathrm{k}$ means cluster and then appoint each sample to the closest centroid. Furthermore calculate means of k clusters. If centroids are unchanged than work is done, other wise skip to the step 2 again and start again.

\section{Morphology}

Morphology can be defined as the study of the forms of things, in particular. The morphology consists of four operations separately applied on images as shown below:

\subsection{Erosion}

Erosion is typically applied to binary images, but there are versions that work on grayscale images [19]. The basic effect of the operator on a binary image is to erode away the boundaries of regions of foreground pixels. Formula for erosion is,

Thus areas of foreground pixels shrink in size, and holes. The study of the forms of things, in particular within those areas becomes larger. In this process we increase the black pixel in the image making, it look thinner. Every object pixel that is touching an background pixel is changed into background pixel.

\subsection{Dilation}

Dilation is one of the operators in the area of mathematical morphology, the other being erosion. It is typically applied to binary images, but there are versions that work on grayscale images. Formula for dilation is

\section{$A \oplus B$}

The basic effect of the operator on a binary image is to gradually enlarge the boundaries of regions of foreground pixels. Thus areas of foreground pixels grow in size while holes within those regions become smaller [20].

In dilation we increase the white pixel in the image making, it look broader. Every background pixel that is touching an object pixel is changed into an object pixel.

\subsection{Opening}

In morphology, opening is the dilation of the erosion of set $\mathrm{A}$ by structuring element $\mathrm{B}$ is,

$$
A \circ B=(A \Theta B) \oplus B
$$

Where $\Theta$ and $\oplus$ denote erosion and dilation, respectively in equation (7). Opening removes small objects from the foreground (usually taken as the dark pixels) of an image, placing them in the background, while closing 
removes small holes in the foreground, changing small islands of background into foreground. These techniques can also be used to find specific shapes in an image [43]. The process of "opening" an image will likely smooth the edges, break narrow block connectors and remove small protrusions from a reference image [20].

\subsection{Closing}

In mathematical morphology, the closing of a set (binary image) $\mathrm{A}$ by a structuring element $\mathrm{B}$ is the erosion of the dilation of that set,

$$
A \bullet B=(A \Theta B) \oplus B
$$

Where $\Theta$ and $\oplus$ denote erosion and dilation, respectively in equation (8). In image processing, closing is together with opening, the basic workhorse of morphological noise removal. Opening removes small objects, while closing removes small holes. In this process we firstly do Dilation and then Erosion. This method is used to remove the extra black pixels from the images [20]. The process of "opening" an image will likely smooth the edges, break narrow block connectors and remove small protrusions from a reference image.

\section{III.}

Experimental Results

Data set comprises of fabric defective images. obtain from denim fabric images official website.
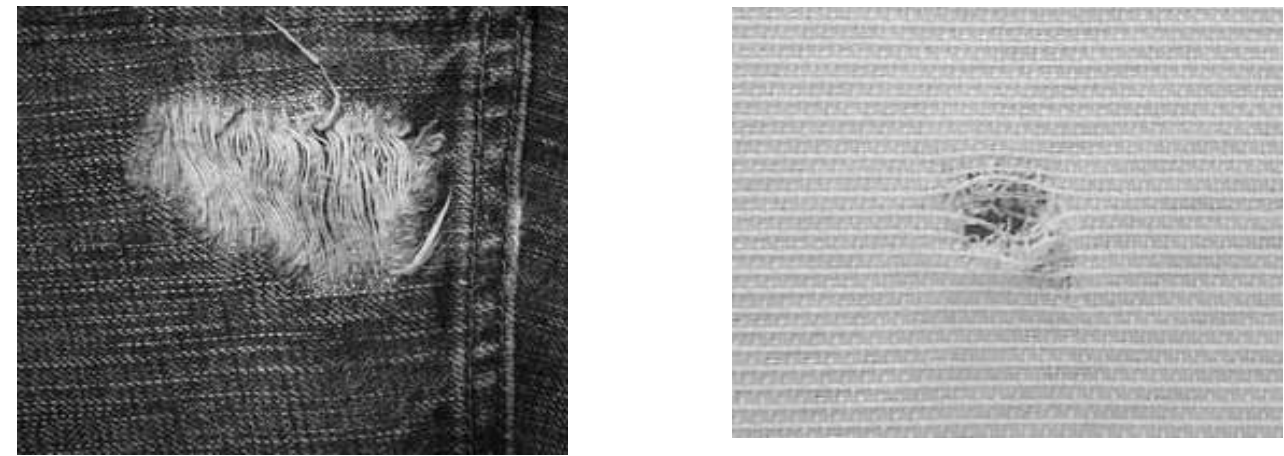

fig.(3.1) jeans image with fabric defect

fig.(3.2) Cotton imagewith defect

fig.(3.3) comprises of different segmented image results of denim jeans image after apply proposed algorithm and morphology techniques

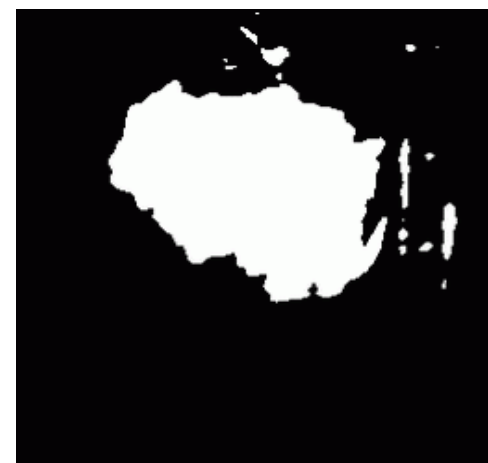

(a)

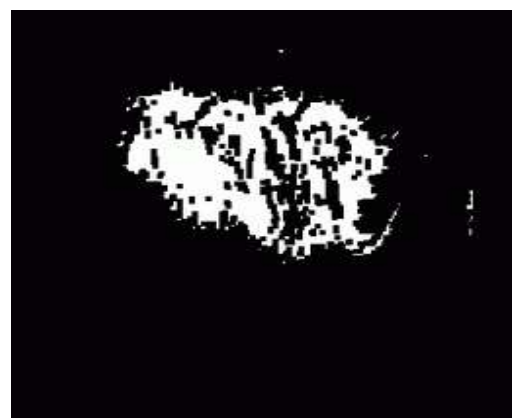

(d)

(e)

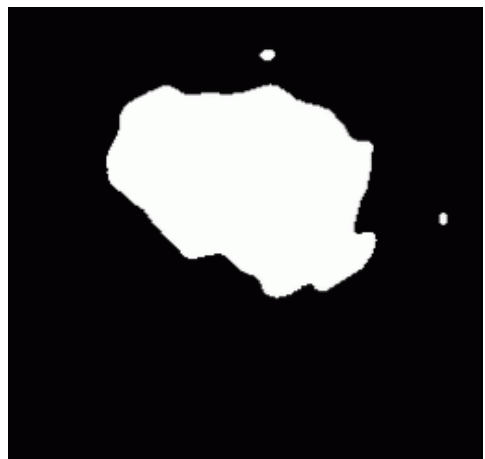

(b)

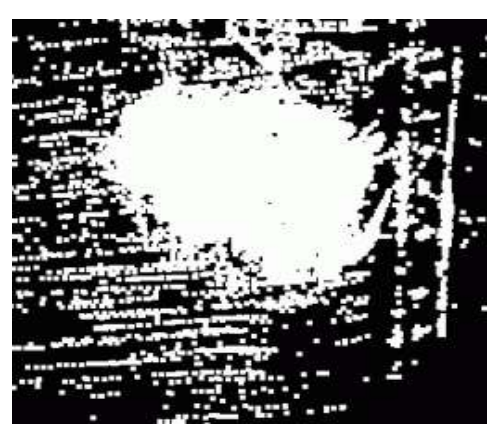

(f)

(c)
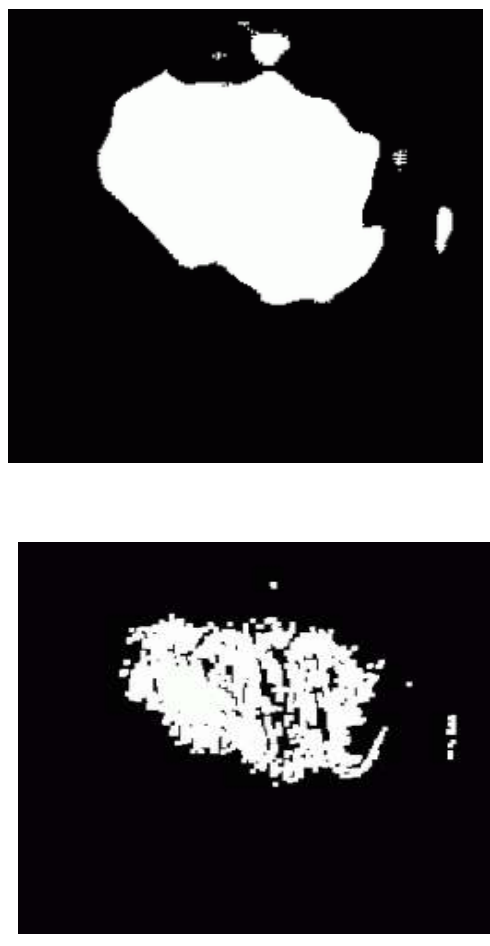


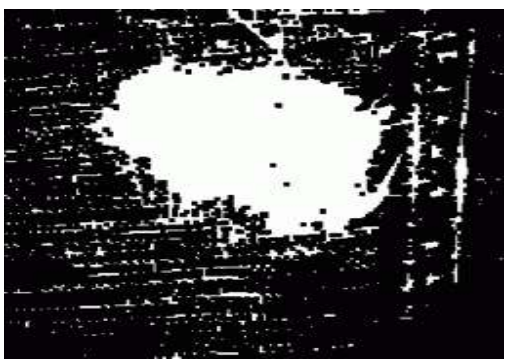

$(\mathrm{g})$

Fig. (3.3)Segmented Results of jean Image (a) $f=0.5$ (b) $b=0.5$ (c) $y=2$ (d) erosion(e)dilation(f)opening(g)closing

fig.(3.4) comprises of different segmented image results of cotton image after apply proposed algorithm and morphology techniques

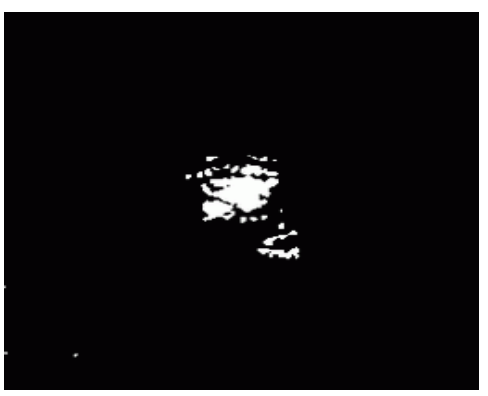

(b)

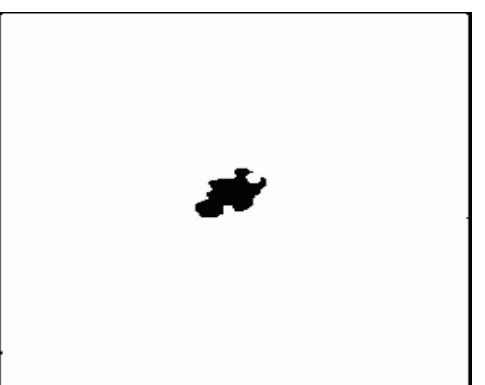

(c)

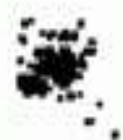

(d) (e)
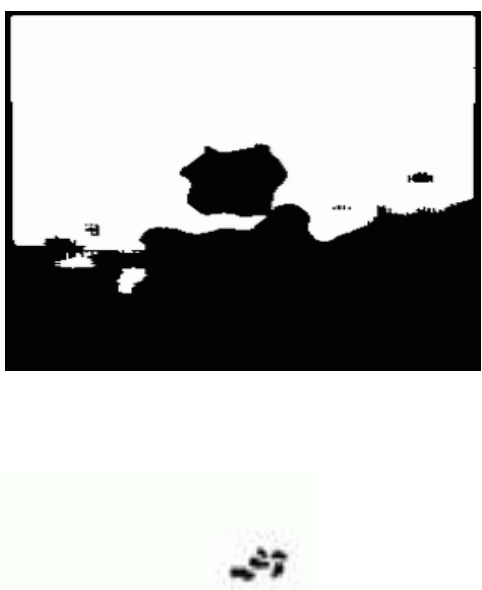

(f)

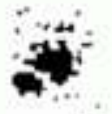

$(\mathrm{g})$

Fig.(3.4)Segmented Results of jean Image (a) $f=1$ (b) $b=1$ (c) $y=0.5(d)$ erosion(e)dilation(f)opening(g)closing

In Table 1 calculate black and white pixels for segmented results of jeans image after apply proposed algorithm and morphology technique.

Table 1: Results for Jeans Image

\begin{tabular}{|l|c|c|c|c|}
\hline \multicolumn{1}{|c|}{ Parameters } & $\begin{array}{c}\text { Original gray scale } \\
\text { area }\end{array}$ & Total no of pixels & $\begin{array}{c}\text { Black pixels \& } \\
\text { \%black pixels }\end{array}$ & $\begin{array}{c}\text { White pixels \& } \\
\% \text { white pixels }\end{array}$ \\
\hline \multicolumn{5}{|c|}{ Gabor filter } \\
\hline $\mathrm{f}=0.5$ & 65536 & 65536 & $51794,79.1 \%$ & $13742,20.9 \%$ \\
\hline $\mathrm{b}=0.5$ & 65536 & 65536 & $52638,80.3 \%$ & $12898,19.7 \%$ \\
\hline $\mathrm{\gamma}=2$ & 65536 & 65536 & $49854,76.1 \%$ & $15682,23.9 \%$ \\
\hline \multicolumn{5}{|c|}{ Morphology } \\
\hline Erosion & 65536 & 65536 & $57158,87.2 \%$ & $8378,12.8 \%$ \\
\hline Dilation & 65536 & 65536 & $30370,46.3 \%$ & $35166,53.7 \%$ \\
\hline Opening & 65536 & 65536 & $54873,83.7 \%$ & $10663,16.3 \%$ \\
\hline Closing & 65536 & 65536 & $42500,64.8 \%$ & $23036,35.2 \%$ \\
\hline
\end{tabular}


In Table 2 measure effective area by dividing segmented area by original image defective area and calculate area error. The segmented area greater or nearly equal to original image defective area will be effective

Table 2: Effective area measurement for Jeans image

\begin{tabular}{|l|c|c|c|}
\hline \multicolumn{1}{|c|}{ Parameters } & $\begin{array}{c}\text { Original image defected area in } \\
\text { pixels }\end{array}$ & $\begin{array}{c}\text { Segmented image } \\
\text { defected area in pixels }\end{array}$ & $\begin{array}{c}\text { Effective } \\
\text { Area=SI/OI }\end{array}$ \\
\hline \multicolumn{4}{|c|}{ Gabor filter } \\
\hline $\mathrm{f}=0.5$ & 12250 & 14742 & 1.2218 \\
\hline $\mathrm{b}=0.5$ & 12250 & 13742 & 1.1217 \\
\hline $\mathrm{Y}=2$ & 12250 & 15800 & 1.2897 \\
\hline \multicolumn{4}{|c|}{ Morphology } \\
\hline Erosion & 3306 & 671 & 0.2029 \\
\hline Dilation & 3306 & 47 & 0.0142 \\
\hline Opening & 3306 & 323 & 0.0977 \\
\hline Closing & 3306 & 120 & 0.0363 \\
\hline
\end{tabular}

Table 3 is about computation of black and white pixels for cotton image

Table 3: Results for Cotton image

\begin{tabular}{|l|c|c|c|c|}
\hline \multicolumn{1}{|c|}{ Parameters } & $\begin{array}{c}\text { Original gray scale } \\
\text { area }\end{array}$ & Total no of pixels & $\begin{array}{c}\text { Black pixels \& } \\
\text { \%black pixels }\end{array}$ & $\begin{array}{c}\text { White pixels } \\
\text { \& } \% \text { white } \\
\text { pixels }\end{array}$ \\
\hline \multicolumn{5}{|c|}{ Gabor filter } \\
\hline $\mathrm{f}=1$ & 65536 & 65536 & $64045,97.7 \%$ & $1491,2.3 \%$ \\
\hline $\mathrm{b}=1$ & 65536 & 65536 & $2180,3.3 \%$ & $63356,96.7 \%$ \\
\hline $\mathrm{y}=0.5$ & 65536 & 65536 & $29233,44.6 \%$ & $36303,55.4 \%$ \\
\hline \multicolumn{5}{|c|}{ Morphology } \\
\hline Erosion & 65536 & 65536 & $57158,87.2 \%$ & $8378,12.8 \%$ \\
\hline Dilation & 65536 & 65536 & $30370,46.3 \%$ & $35166,53.7 \%$ \\
\hline Opening & 65536 & 65536 & $54873,83.7 \%$ & $10663,16.3 \%$ \\
\hline Closing & 65536 & 65536 & $42500,64.8 \%$ & $23036,35.2 \%$ \\
\hline
\end{tabular}

In Table 4 effective area have been measured for cotton image by dividing segmented areas by original image defective area.

Table 4: Effective area measurement for Cotton image

\begin{tabular}{|l|c|c|c|}
\hline \multicolumn{1}{|c|}{ Parameters } & $\begin{array}{c}\text { Original image defected area in } \\
\text { pixels }\end{array}$ & $\begin{array}{c}\text { Segmented image } \\
\text { defected area in pixels }\end{array}$ & $\begin{array}{c}\text { Effective } \\
\text { Area=SI/OI }\end{array}$ \\
\hline \multicolumn{3}{|c|}{ Gabor filter } \\
\hline $\mathrm{f}=1$ & 3306 & 1491 & 0.4510 \\
\hline $\mathrm{b}=1$ & 3306 & 2180 & 0.6594 \\
\hline $\mathrm{V}=0.5$ & 3306 & 29233 & 0.8424 \\
\hline \multicolumn{3}{|l|}{ Morphology } \\
\hline Erosion & $3306 \quad 3306$ & 47 & 0.0142 \\
\hline Dilation & 3306 & 323 & 0.0977 \\
\hline Opening & 3306 & 120 & 0.0363 \\
\hline Closing & & & \\
\hline
\end{tabular}

Comparison of proposed method and morphology technique have been shown in figure 3.5 


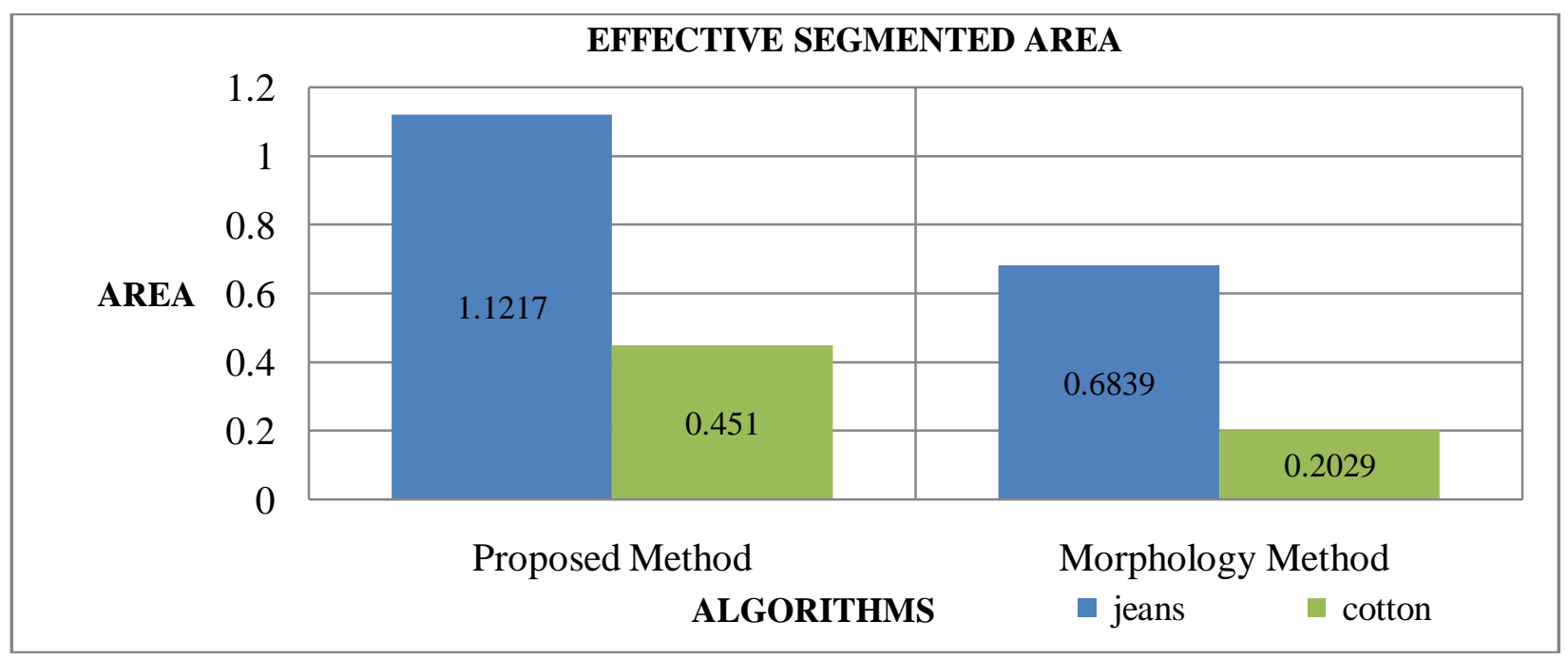

Fig. (3.5) Graphical comparison of Gabor filter and Morphology technique results

\section{Conclusion}

In this paper proposed algorithm based on 2-D Gabor filter can be adopted for defect detection in textile images tuned at different parameters and morphology technique have been used for defect segmentation. Experiments on two different images have been done. The experimental results have shown that segmented area of proposed method is much better than morphology technique. The effective area measurement of proposed method is better than morphology technique. We use proposed method presented in this paper for visual inspection in textile industry in which camera is attached with PC. When cotton roll move than camera move on the roll of the cloth and detect defect and send this information to pc and then pc measures and calculate the defective area and other properties related to that fabric defect. This method is also fruit full in other defect detection fields like tumor detection, iris detection, finger print detection, license plate detection etc. This paper also give help about segmentation of various images in textile industry, medical imaging, iris recognition.

\section{References}

[1] R. C. Gonzalez, R. E.Woods, Digital Image Processing, Pearson Education 2002.

[2] D. Gabor, “Theory of Communication”, The Journal of the Institution of Electrical Engineers, Pp.429-445, 1946.

[3] P. Brodatz, "Textures: A Photographic Album for Artists and Designers", Dover, 1966.

[4] Google scholar. 2009 (February), Data download from http://scholar.google.com.

[5] Baldi, G Hatefield," Dna microarrays and gene expression", Cambridge University Press, 2002.

[6] A.K. Jain, "Data Clustering: 50 Years Beyond K-Means", Pattern Recognition Letters, Vol. 31, No. 8, pp. 651-666, 2010

[7] Bhoi,Khagswar and Dipesh Kumar solanki “Texture Segmentation Using Optimal GaborFilter,"BTech thesis, National Institute of Technology Rourkela,Rourkela-769 008, Orissa,India,2011.

[8] AthmanBouguettaya "On Line Clustering", IEEE Transaction on Knowledge and Data Engineering Volume 8, No. 2, April 1996.

[9] Bruce Moxon "Defining Data Mining, TheHows and Whys of Data Mining, and How It Differs From Other AnalyticalTechniques" Online Addition of DBMS Data WarehouseSupplement, August 1996

[10] A. K. Jain, F. Farrokhnia, ”Unsupervised texture segmentation using Gabor filters", Patternrecognition, vol. 24, no. 12, pp.11671186,1991

[11] X. Xie, "A review of recent advances in surface defect detection using texture analysistechniques", Electronic Letters on ComputerVision and Image Analysis, 7:1-22, 2008.

[12] Mahajan P.M, Kolhe S.R and Patel P.M,"A review of automatic fabric defect detectiontechniques", advances in computationalresearch, vol 1, PP-18-29, issue 2, 2009.

[13] Ajay kumar,"Computer vision based Fabric defect detection:A survey",IEEE Transactions onIndustrial Electronics, Vol. 55, No1, pp. 348-363,4 january2008.

[14] K Hammouda and E. Jernigan, "Texture segmentation using Gabor filter" tech. rep, University of Waterloo, Canada.[15]JianguoZhang,Tieniu Tan and Li Ma, "Invariant texture segmentation via circular Gaborfilter", Proceedings of the 16th IAPRInternational Conference on Pattern Recognition (ICPR), Vol II, pp. 901-904, 2002.

[16] N. Petkov, M.B. Wieling,'Gabor filter for image processing and computer vision", Department of computing science and intelligentsystem, University of Groningen, Holland.

[17] http://www.richelbilderbeek.nl/ToolGaborFilter.html

[18] http://home.dei.polimi.it/matteucc/Clustering/tutorial_html

[19] SatishPawar and V. K. Banga, "Morphology Approach in Image Processing", International Conference on Intelligent Computational Systems (ICICS'2012), Jan. 7-8, 2012

[20] K.L. Mak, P. Peng and K.F.C. Yiu,"Fabric defect detection using morphological filters",Image and Vision Computing , Volume: 27, pp.1585-1592, July 2009.

[21] AsitDatta and Jayanta Kumar Chandra, "Detection of Defects in Fabric by Morphological Image Processing", Woven Fabric Engineering, PolonaDobnikDubrovski (Ed.), ISBN: 978-953-307-194-7, 2010. 